Ansaidioo唯

And

$A^{\text {rthritis }}$

INDICATIONS: Acute and long term treatment of signs and symptoms of rheumatoid arthritis and osteoarthritis.

CONTRAINDICATIONS: Hypersensitivity to ANSAID, or if aspirin or any other nonsteroidal antiinflammatory agent induces asthma, urticaria or other allergic type reactions. Fatal asthmatic reactions have been reported in such patients.

WARNINGS: Gastrointestinal effects: Risk of Gl ulcerations, bleeding and perforation with nonsteroidal anti-inflammatory therapy: Serious GI toxicity can occur at any time, with or without warning symptoms, during chronic treatment. The occurrence is about $1 \%$ after 3-6 months, 2-4\% after a year. Patients should be informed of signs and symptoms of serious Gl toxicity and what to do if it occurs. No subset of patients not at risk has been identified. Prior history of serious Gl events and other risk factors of peptic ulcer disease, e.g. alcoholism, smoking, etc., have been associated with increased risk. The elderly and debilitated tolerate ulceration and bleeding less well. Higher doses probably carry a greater risk. Gl ulceration and bleeding can occur without warning symptoms and chronically treated patients should be followed.

PRECAUTIONS: Patients with impaired renal or hepatic function: Use ANSAID and similar agents cautiously. Pharmacokinetics have not been studied in patients with decreased liver function.

Renal Effects: Rats develop renal papillary necrosis at dosages equivalent to human therapeutic levels, as do monkeys given 20-40 times the human dose. In clinical studies of ANSAID, kidney function tests were done monthly and renal effects were similar to those seen with other nonsteroidal anti-inflammatory drugs. A second form of renal toxicity has been seen in patients with prerenal conditions that reduce renal blood flow or blood volume. A nonsteroidal anti-inflammatory drug may cause dose-dependent reduction in prostaglandin formation and precipitate overt renal decompensation. Patients at greatest risk are those with impaired renal or hepatic function heart failure, those taking diuretics or the elderly. Drug discontinuation usually leads to recovery. Patients at high risk on chronic treatment should have rena discon function monito ( or symptoms. Flurbiproten is excreted by the kidneys and pharmacokinetics are changed by renal failure so patients with renal failure should be monitored and may require a reduction of dosage to avoid accumulation of flurbiprofen metabolites.

Liver tests: Borderline elevations of liver function tests may occur in up to $15 \%$ of patients, and may progress, remain unchanged or disappear with continued treatment. Patients with signs and/or symptoms or with an abnormal liver function test should be evaluated further.

Anemia: Patients treated long term who have initial hemoglobin values under $10 \mathrm{~g} / \mathrm{dL}$, should have periodic hemoglobin values.

Fluid retention and edema: Fluid retention and edema have been reported so use ANSAID with caution in patients with conditions such as cardiac decompensation or hypertension.

Vision Changes: Blurred and/or diminished vision has been reported. Patients with eye complaints should have periodic ophthalmologic exams.

Effect on platelets and coagulation: Platelet aggregation is inhibited and bleeding time prolonged, patients who may be adversely affected should be carefully observed.

Information for patients: Physicians and patients may wish to discuss potential risks and likely benefits.

Drug Interactions: Anticoagulants: Bleeding parameters are affected, clinical bleeding has been reported. Aspirin. Flurbiprofen levels were $50 \%$ lower. Concurrent use is not recommended Betareported. Aspirin: Flurbiprofen levels were $50 \%$ lower. Concurrent use is not recommended. Betaadrenergic Blockers: Pharmacokinetics and heart rate reduction are not affected, hypotensive effect of propranolol but not atenolol was attenuated. Cimetidine, Ranitidine: Cimetidine causes a 13\% furosemide or thiazides should be closely observed to make sure the desired effect is obtained.

Carcinogenesis, mutagenesis, impairment of fertility: No evidence.

Teratogenic effects: Pregnancy category B: No effect in animals. Not recommended for use in pregnancy.

Labor and delivery, nursing mothers, pediatric use: Use is not recommended

ADVERSE REACTIONS: $9.4 \%$ of 4123 patients dropped out of studies because of an a.d.r. Incidence $>1 \%$. Gastrointestinal: Dyspepsia* diarrhea* abdominal pain* nausea* constipation, GI bleeding flatulence, elevated liver enzymes and vomiting central nervous system: Headache* "stimulation" (eg. anxiety, insomnia, reflexes increased, tremor) and "inhibition" (eg. amnesia, asthenia, somnolence, anxiety, insomnia, reflexes increased, tremor) and "inhibition" (e.g. amnesia, asthenia, somnolence, malaise and depression). Respiratory: Rhinitis. Dermatologic: Rash. Special senses: Dizziness, tionitus and changes in vision. Genitourinary: Signs and symptoms suggest

* Reaction in 3 to $7 \%$ of patients.

Incidence <1\% (Causal relationship probable): Gastrointestinal: Peptic ulcer disease (See Warnings), gastritis, bloody diarrhea, stomatitis, esophageal disease, hematemesis and hepatitis, cholestatic and non-cholestatic jaundice. Central nervous system: Ataxia, cerebrovascular ischemia, confusion, paresthesia and twitching Hematologic: Decrease in hemoglobin and hematocrit, iron deficiency anemia, leukopenia, eosinophilia and ecchymosis, thrombocytopenia, hemolytic anemia and aplastic anemia. (See Precautions) Respiratory: Asthma and epistaxis. Dermatologic: Angioederna, urticaria eczema and pruritus; photosensitivity, toxic epidermal necrolysis and exfoliative dermatitis. Special senses: Coniunctivitis and parosmia. Genitourinary: Hematuria and impairment of renal function interstitial nephritis Body as a whole: Anaphylactic reactions, chills, fever. Metabolic/Nutritional: Hyperuricemia. Cardiovascular: Heart failure, hypertension, vascular disease and vasodilatation.

Incidence <1\% (Causal relationship unknown): Gastrointestinal: Periodontal abscess, appetite changes, cholecystitis and dry mouth. CNS: Convulsion, meningitis, hypertonia, cerebrovascular accident, emotional lability and subarachnoid hemorrhage. Hematologic: Lymphadenopathy, Respiratory Bronchitis, laryngitis, dyspnea pulmonary embolism, pulmonary infarct, hyperventilation Dermatologic: Alopecia, nail disorder, herpes, dry skin and sweating. Special senses: Ear disease, corneal 10logic. Alopecia, nail disorder, herpes, dry skin and sweating. Special senses. opacity, glaucoma, retrobulbar neuritis, change in taste, transient hearing loss, retinal hemorrmage. Genitourinary: Menstrual disturbances, vaginal and uterine hemorrhage, vulvovaginitis, prostate disease. Metabolic/nutritional: Hyperkalemia. Carction.
dial infarction. Musculoskeletal: Myasthenia.

dial infarction. Musculoskeleta: Myasthenia. cheumatoid arthritis has been with tid or qid dosage). Dose should be tailored to severity of symptoms and patient response.

Store at controlled room temperature $\left(15-30^{\circ} \mathrm{C}\right)$.

Federal law prohibits dispensing without a prescription

\section{What will it take to reduce the incidence of breast cancer?}

The latest Centers for Disease Control (CDC) figures indicate a continuing rise in the incidence of breast cancer. Between 1979 and 1986 , the number of cases increased $24 \%$. During this same period, breast cancer mortality rose approximately $5 \%$, from $31.1 \%$ to $32.8 \%$ per 100,000 women. In fact, 40,000 women died of breast cancer in 1986 alone. Based on estimates from the American Cancer Society, 1 woman in 10 was expected to develop breast cancer in 1989.

See related article beginning on page 47

Despite research discoveries, this mortality rate has not decreased for the past several decades. This fact proves particularly discouraging because the National Cancer Institute estimates a $50 \%$ reduction in mortality when preventive measures are taken and early diagnosis is made.

As an example of one preventive measure, the most recent epidemiologic trends suggest a relationship between a high fat diet and breast cancer. Although the final word is not yet in, it seems prudent for physicians to recommend their patients reduce their total dietary fat intake to $30 \%$ of calories. Such a precaution may protect not only against coronary artery disease, but breast cancer as well.

Yet these and other preventive methods and technologies are effective only when backed by an aggressive educational campaign directed toward the public and physicians. These educational efforts should emphasize the importance of breast self-examination (BSE). Women themselves detect more than $90 \%$ of breast cancers through monthly BSE. There-

(continued on page 26 ) 


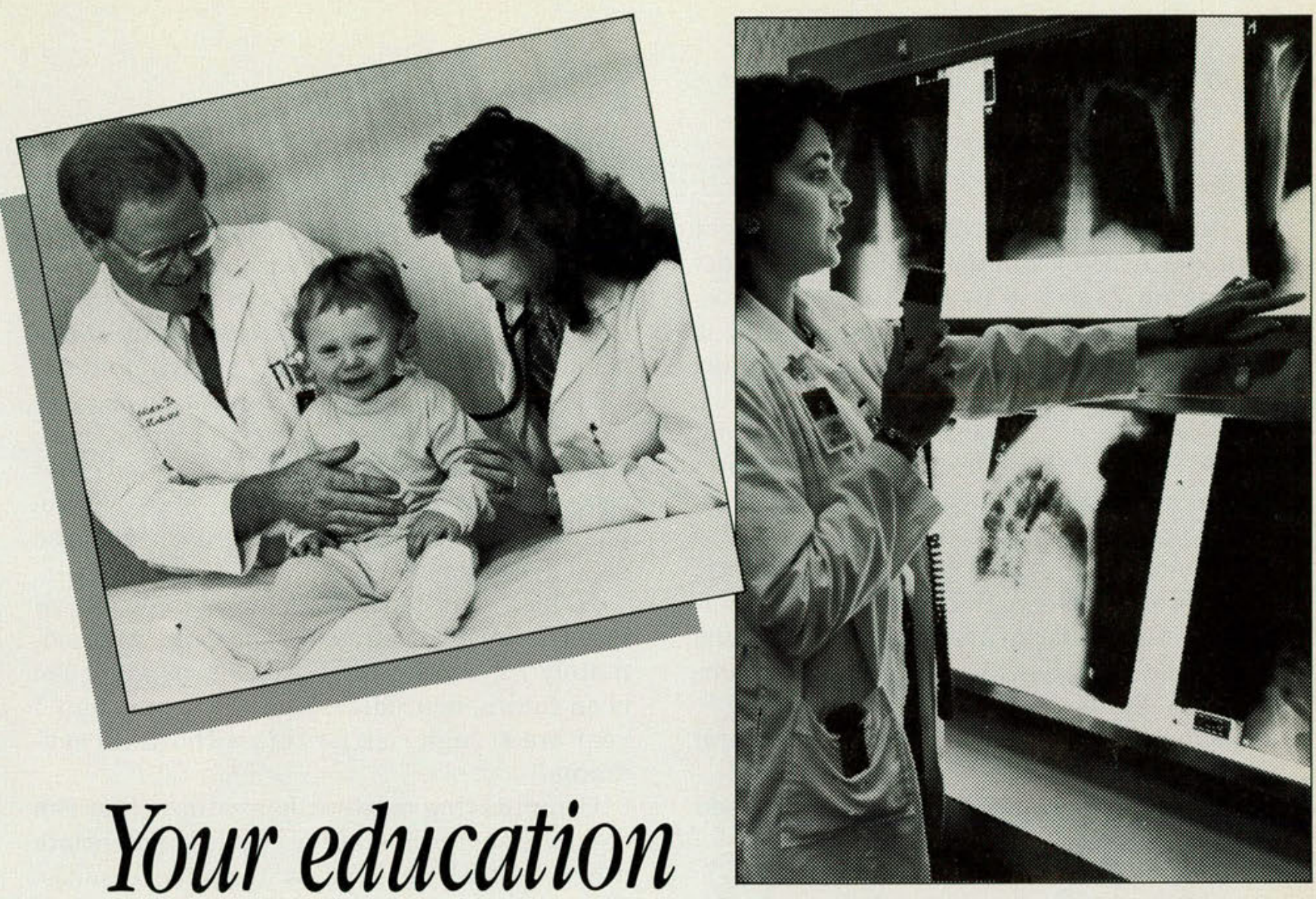

\section{is important to us!}

Left Pboto: Don Middleton, D.O.

Resident, Tracy Overton, D.O.,

Resident and "small" patient at FOH.

Right Pboto: Sucbeta Kulkarni, D.O., Intern

"Flint Osteopathic Hospital has provided acute and general health care for the Flint community for 50 years. The hospital is affiliated with Michigan State University and provides a full range of medical, surgical, emergency, and intensive care services. All medical and surgical subspecialties are covered. At FOH the intern and resident receives training in both outpatient ambulatory care as well as inpatient practices

FOH, with 359 beds, is the largest osteopathic hospital in Michigan offering intern and residency training programs for osteopathic physicians. The medical education program is designed to provide a structured curriculum and experience in diagnosis and treatment. Morning reports and guest physician lectures occur daily. Reading lists and objectives have been developed for each service. A monthly journal club is conducted by each clinical department. EKG conferences are scheduled twice monthly. The FOH Congdon Lecture Series brings both M.D. and D.O. physicians to the hospital each month. Prominent practitioners, representative of both medical communities, share expertise in research findings during these monthly, day-long seminar presentations.

Ambulatory clinics have been established and provide longitudinal continuity training for interns and residents. Both traditional and alternative track internships are available at $\mathrm{FOH}$. The hospital is a charter member of the Consortium of Osteopathic Graduate Medical Education and Training (COGMET) in association with Michigan State University."

\section{Residencies}

- Anesthesia

- Family Practice

- Gastroenterology

- Internal Medicine

- Obstetrics/Gynecology

- Ophthalmology

- Orthopedics

- Otorhinolaryngology

- Pathology

- Pulmonary

- Radiology

- Surgery

- Urology

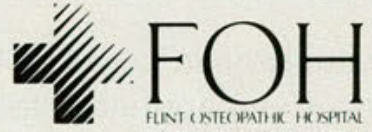

3921 Beecher Rd.

Flint, MI 48532

\section{Where family matters}

\section{Fellowships/Subspecialty} Residencies

- Medical Diseases of the Chest - Gastroenterology

One-year rotating internships Student externships

Christopher T. Meyer, D.O. Vice President of Medical Education

Dennis V. DeSimone, D.O. Director of Medical Education

(313) $762-4707$ 
fore, every woman in her 20 s should be taught the proper way to examine her breasts. By developing an expertise in BSE, a woman fosters self-confidence in her technique and is more likely to practice BSE on a regular monthly basis.

A yearly physician palpatory examination, coupled with mammography, complements BSE. In particular, the value of mammography cannot be understated.

Currently, the American Cancer Society, the American Osteopathic College of Radiology, and nine other healthcare organizations recommend the following guidelines for mammography for asymptomatic women:

- baseline mammogram between ages 35 and 40

- a mammogram every 1 to 2 years between ages 40 and 50

- an annual mammogram after age 50

Patients who were previously treated for breast cancer or who have a family history of premenopausal breast cancer (mother or sister) should undergo annual mammography at an earlier age.

Such a comprehensive approach remains a crucial component in our efforts to save women's lives.

THOMAS WESLEY ALLEN, DO

\section{Education-not legislation- resolves benzodiazepine abuse}

Our society has an insatiable need to pass laws regulating our behavior. Even applied reason does not deter our desire for simple solutions to complex problems. Such is the case with the current controversy surrounding benzodiazepines.
For almost 30 years, benzodiazepines have been prescribed to treat a variety of conditions. As with all drugs, certain potentially inherent risks exist. Recently, benzodiazepines have been linked with cognitive dysfunction in the elderly. Likewise, anterograde amnesia can occur when benzodiazepines are taken with alcohol. Physicians are aware of these and other risks associated with this family of psychotropic drugs. These include long-term use and drug dependency.

In fact, long-term benzodiazepine use now has a defined withdrawal syndrome. Approximately 1.2 to 2.2 million Americans who have been taking benzodiazepines for more than 1 year are at high risk for this withdrawal syndrome.

Complicating matters, benzodiazepines can create a euphoric state as well as potentiate other central nervous system depressants. Thus, this legal drug is being used illegally as a "street drug." Such abuse has occurred, in part, because some physicians have been less-than meticulous in prescribing and monitoring patients' use of benzodiazepines.

Private, professional, and government organizations have expressed their concerns regarding such abuse. Most sought to solve the problem by educating physicians and the public as to the appropriate use of this drug.

Local legislators have moved to reclassify this drug family or require a triplicate prescription for benzodiazepines. New York State took the latter approach. Effective Jan 1, 1989, all physicians who write benzodiazepine prescriptions in that state must do so on a registered form, which also limits the number of refills. Purchased from the state at a cost of $\$ 6.25$ per pad, these prescription pads are sold illegally on the streets of New York City for $\$ 100$ to $\$ 125$.

During the first weeks of New York's program, the number of benzodiazepine prescrip-

(continued on page 30 ) 